\title{
Low Density Polyethylene (LDPE) Waste Plastic Transformation into Renewable Heavy Fuel Using Thermal Cracking
}

\author{
Moinuddin Sarker*, Mohammad Mamunor Ras hid, Muhammad Sadikur Rahman
}

Natural State Research, Inc. Department of Research and Development, 37 Brown House Road (2 ${ }^{\text {nd }}$ Floor), Stamford, CT 06902, USA

\begin{abstract}
Low density polyethylene (LDPE) waste plastic was transformed into heavy fuel by using fractional distillation column process with liquefaction process. For experimental purpose two types of temperature profiles were used one for LDPE waste plastic liquefaction temperature and another one for fractional distillation column temperature. In this experiment liquefaction temperature ranges $100-420^{\circ} \mathrm{C}$ and fractional distillation temperature ranges $340-365^{\circ} \mathrm{C}$. LDPE waste plastic to heavy fuel production was performed without adding any kind of catalyst in presence of oxygen and without vacuum system. Different types of analytical equipment were used for produced fuel analysis purposed such as GC/MS, FT-IR and DSC. By using GC/MS analysis result indicates that LDPE waste plastic to produced heavy fuel hydrocarbon chain range $C_{5}$ to $C_{28}$ and light gas are also present and hydrocarbon range is $C_{1}-C_{4}$. FT-IR (Spectrum 100) analysis results indicate that produced fuel functional group has bands energy which is reflecting with calorific value. By using DSC analysis result showed produced fuel delta $\mathrm{H}$ value or enthalpy value. Produce fuel can be use for heavy equipment internal combustion engine or electricity generation or feed for feed stock refinery process.
\end{abstract}

Keywords Polyethylene, Waste Plastic, LDPE, Thermal, Renewable, Fuel, Hydrocarbon, GC/MS

\section{Introduction}

The decomposition of waste plastics into fuel represents a sustainable way for the recovery of the organic content of the polymeric waste and also preserves valuable petroleum resources in addition to protecting the environment[1]. Municipal waste plastic represents about $8 \mathrm{wt} \%$ of the municipal solid waste and it generally consists of mixture of different kind of plastics: $40.5 \mathrm{wt} \%$ HDPE (High Density Polyethylene) and LLDPE/LDPE (Linear/Low Density Polyethylene ), 19.6 wt\% PP (Polypropylene), 11.9 wt $\%$ EPS PS/ (Expandable Polystyrene / Polystyrene), $10.7 \mathrm{wt} \%$ PVC (Polyvinyl Chloride), $8.1 \mathrm{wt} \%$ PET (Polyethylene terephthalate), and about 5 wt \% ABS (Acrylonitrile Butadien Styrene) and $4.2 \mathrm{wt} \%$ other polymers [2]. This waste is difficult to be treated or recycled due to its complex nature and composition, structural deterioration of the polymeric components and the contamination with various organic, inorganic or biological residues. Pyrolysis is one of the best methods to recover the material and energy from polymer waste, as only about $10 \%$ of the

\footnotetext{
* Corresponding author:

msarker@naturalstat eres earch.com (Moinuddin Sarker)

Published online at http://journal.sapub.org/env

Copyright (C) 2012 Scientific \& Academic Publishing. All Rights Reserved
}

energy content of the waste plastic is used to convert the scrap into valuable hydrocarbon products[3]. The conversion of waste plastics into fuel represents a sustainable way for the recovery of the organic content of the polymeric waste and also preserves valuable petroleum resources in addition to protecting the environment[4]. The worlds limited reserve of coal, crude oil and natural gas places a great pressure on mankind to preserve its existing non-renewable materials. Among the various recycling methods for the waste plastics, the feedstock recycling has been found to be a promising technique. There are a lot of research[4-15] in progress on the pyrolysis and utilization of pyrolysis products for various applications.

Thermal degradation of polymer mixtures is more compl ex than the degradation of single poly mers. Interactions can appear at high temperatures during decomposition in the polymer bulk, between the components of the mixture and the low molecular weight products and free radicals that are formed by the scission of the polymeric chains. Due to these interactions, some polymers can affect the quality of pyrolysis products even they are in small amount in polymer mixture. This is of very high interest especially when the pyrolysis oils have to pass some quality standards for use as feedstock or fuels[16]. ABS with flame retardant PVC and PET are heteroatom containing polymers that create problems during thermal decomposition because bromine, chlorine and nitrogen can remain as organic 
compounds in pyrolysis oils or can produce acid or toxic gases such as $\mathrm{HCl}, \mathrm{HBr}$, and $\mathrm{HCN}, \mathrm{NH}_{3}$ or polyhalogenated dibenzodioxins and dibenzofurans [17, 18]. Therefore, the amount of these compounds has to be decreased as much as possible. Thermal degradation of ABS gives oils with high content of benzene derivatives (toluene, ethylbenzene, styrene, cumene, and $\alpha$-methylstyrene) but also containing significant amount of organic nitrogen as aliphatic and arom atic nitriles or nitrogen containing heterocyclic compounds. The amount and distribution of these compounds strongly depends on the thermal or catalytic conditions used for degradation $[19,20]$.

\section{Materials and Method}

The raw materials were collection from Stamford city Wal-Mart store. Collected raw material comes with other plastic also such as LDPE (Low Density Polyethylene), HDPE (High Density Polyethylene), PP (Polypropylene) and PS (Polystyrene). All plastics separated by manually from mixed plastics. LDPE waste plastic washed with soap and water by using sink because LDPE waste plastic was shopping or packaging bag and color was various types. Then washout LDPE waste plastic dried into room temperature by putting on the laboratory floor. After that LDPE waste plastic cut into small pieces manually by using scissors. Small pieces LDPE waste plastic shopping bags were manually transferred into reactor chamber. Stainless steel reactor was setup under laboratory fume hood without vacuum system and in presence of oxygen and it was fully closed system. Reactor was heated by electrical power and reactor capable temperature range was $25-500^{\circ} \mathrm{C}$. For experiment purpose temperature rang was $100-420^{\circ} \mathrm{C}$. Experimental set up were describe in Figure 1 and all number describe such as $1=$ Low density polyethylene waste plastic, $2=$ Steel reactor, $3=$ Fractional distillation column, $4=1^{\text {st }}$ fraction temperature, $5=2^{\text {nd }}$ fraction temperature, $6=3^{\text {rd }}$ fraction temperature, $7=4^{\text {th }}$ fraction temperature, $8=5^{\text {th }}$ fraction temperature, $9=$ light gas cleaning system, $10=1^{\text {st }}$ fraction fuel collection tank, $11=2^{\text {nd }}$ fraction fuel collection tank, $12=3^{\text {rd }}$ fraction fuel collection tank, $13=4^{\text {th }}$ fraction fuel collection tank, $14=5^{\text {th }}$ fraction fuel collection tank, $15=$ s mall pump, $16=$ Teflon bag for light gas storage. In this experiment LDPE waste plastic was used only $1000 \mathrm{gm}(1 \mathrm{~kg})$ and this experiment did not use any kind of chemical or catalyst. After setup the experiment started temperature was $100^{\circ} \mathrm{C}$ and temperature increased up $420^{\circ} \mathrm{C}$ gradually. Initial raw material LDPE plastic melting point temperature $120^{\circ} \mathrm{C}$ and experimental temperature was $100^{\circ} \mathrm{C}$. Fractional colu mn temperature was setup by using electrical coil and temperature profile was for $1^{\text {st }}$ fractional at $40-65^{\circ} \mathrm{C}, 2^{\text {nd }}$ fractional at $110-135^{\circ} \mathrm{C}, 3^{\text {rd }}$ fractional at $180-205^{\circ} \mathrm{C}, 4^{\text {th }}$ fractional at $260-285^{\circ} \mathrm{C}$ and $5^{\text {th }}$ fractional at $340-365^{\circ} \mathrm{C}$. In the experiment the main goal was to collect heavy fuel from LDPE waste plastic. By using this technology waste plastic converted into liquefaction from $100-420{ }^{\circ} \mathrm{C}$ and fractional column was setup for collection heavy fuel and other grade fuels. LDPE waste plastic melt due to heat applies then turn into liquid slurry when temperature increased gradually. Liquid slurry turn into vapor then vapor passed through into fractional distillation column surface and at the end vapor transform to fuel and the product was collected based on different fractional boiling point range wise. Negative boiling point vapor come out as light gas and this light gas hydrocarbon mixture of methane, ethane, propane and butane. Rest of all fractional fuels was collected according to their boiling point range like minimum temperature to maximum temperature wise such as $1^{\text {st }}$ fractional to $5^{\text {th }}$ fractional fuel. In diagram number 14 is $5^{\text {th }}$ fractional fuel or heavy fuel and this fractional tower temperature was $340-365^{\circ} \mathrm{C}$. During LDPE to heavy fuel production process didn't escape or leakage any gases materials into the environment and capture light gas was purified by using alkali solution then light gas transfer into Teflon bag by using small pump. Collected produced fuel was purified by using RCI technology provided RCI fuel purifier with centrifugal force and micron filter. In this experimental process heavy fuel production percentage was $18.1 \%$ and other fractional fuels was $73.9 \%$. Remaining solid black residue was $3 \%$ and light gas was only $5 \%$ from total $1000 \mathrm{gm}$ of LDPE waste plastic. In mass balance calculation from $1000 \mathrm{gm}$ of LDPE waste plastic to heavy fuel conversion rate was $181 \mathrm{gm}$ sample, rest of other grade such as gasoline, naphtha, aviation and diesel grade fuels conversion is total $739 \mathrm{gm}$ sample, light gas generated fro $\mathrm{m} 1000 \mathrm{gm}$ waste plastic to $50 \mathrm{gm}$ sample and finally left over residue was $30 \mathrm{gm}$ from total sample. Residue has also good Btu value and this Btu value more than 5000/lb. Residue could be used as substantial coal, roof carpeting or road carpeting. Produced fuel density is $0.81 \mathrm{~g} / \mathrm{ml}$. Total experiment runtime was 5-6 hours and input electricity was $7.89 \mathrm{kWh}$ according to the laboratory scale. In this experimental process produced light gas could be use for reactor heating purposes and that will significantly reduce the production cost and increase the back up electricity stock.

\section{Results and Discussion}

Perkin Elmer GC/MS (Gas Chromatography and Mass Spectrometer) analysis of $5^{\text {th }}$ fractional fuel/ heavy fuel (Figure 2 and Table 1) hydrocarbon compound list is analysed based on their peak intensity. GC/MS chromatogram analysis is showing higher concentration level peak intensity. This fuel's fractional temperature is $340-365{ }^{\circ} \mathrm{C}$. This fuel is similar to fuel oil category. Chro matogram analysis starting compound is Cyclopropane, ethyl- $\left(\mathrm{C}_{5} \mathrm{H}_{10}\right)$ at retention time is 1.91 minutes. From data table (Table1) we saw all hydrocarbon compounds are straight chain hydrocarbon compounds and some are branch chain hydrocarbon compounds are as well. From the fuel we found alkane group and alkene group compound. Long 
chain hydrocarbon compound showing at retention time 28.45 minutes and compound is Octacosane $\left(\mathrm{C}_{28} \mathrm{H}_{58}\right)$ and molecular weight is 394. In the fuel all hydrocarbon compounds contains heavy hydrocarbon and their derivatives as well as hydrocarbon range is $\mathrm{C}_{5}-\mathrm{C}_{28}$.In the detail analysis prospects maximum compounds are mention from the analysis result index. Such as in detail analysis according to the retention 1.95 and trace mass 42, compound is Pentane $\left(\mathrm{C}_{5} \mathrm{H}_{10}\right)$, retention time 2.53 and trace mass 41, compound is Cyclopropane,1-ethyl-2-methyl -,cis- $\left(\mathrm{C}_{6} \mathrm{H}_{12}\right)$, retention time 2.61 and trace mass, compound is Hexane $\left(\mathrm{C}_{6} \mathrm{H}_{12}\right)$, retention time 3.65 and trace mass 41 ,compound is 1 -Heptene $\left(\mathrm{C}_{7} \mathrm{H}_{14}\right)$, retention time 3.78 and trace mass 41 , compound is Heptane $\left(\mathrm{C}_{7} \mathrm{H}_{16}\right)$,retention time 5.19 and trace mass 41 , compound is 1-Octene $\left(\mathrm{C}_{8} \mathrm{H}_{16}\right)$, retention time 5.35 and trace mass 43 , compound is Octane $\left(\mathrm{C}_{8} \mathrm{H}_{18}\right)$, retention time 6.92 and trace mass 41 ,compound is 1 -Nonene $\left(\mathrm{C}_{9} \mathrm{H}_{18}\right)$, retention time 7.07 and trace mass 43,compound is Nonane $\left(\mathrm{C}_{9} \mathrm{H}_{20}\right)$, retention time 8.65 and trace mass 41 ,compound is 1-Decene $\left(\mathrm{C}_{10} \mathrm{H}_{20}\right)$,retention time 8.79 and trace mass 43 , compound is compound is Decane $\left(\mathrm{C}_{10} \mathrm{H}_{22}\right)$, retention time 10.30 and trace mass 43, compound is 1-Undecene $\left(\mathrm{C}_{11} \mathrm{H}_{22}\right)$, retention time 10.43 and trace mass 43 , compound is Undecane $\left(\mathrm{C}_{11} \mathrm{H}_{24}\right)$,retention time 11.85 and trace mass 41 , compound is 1 -Decene $\left(\mathrm{C}_{10} \mathrm{H}_{24}\right)$, retention time 11.98 and trace mass 43 , compound is Dodecane $\left(\mathrm{C}_{12} \mathrm{H}_{26}\right)$, retention time 13.32 and trace mass 41 , compound is 1-Tridecane $\left(\mathrm{C}_{13} \mathrm{H}_{28}\right)$, retention time 13.43 and trace mass 43 , compound is Tridecane $\left(\mathrm{C}_{13} \mathrm{H}_{28}\right)$, retention time 14.70 and trace mass 41 , compound is
1-Tetradecene $\left(\mathrm{C}_{14} \mathrm{H}_{28}\right)$, retention time 14.81 and trace mass 43 , compound is Tetradecane $\left(\mathrm{C}_{14} \mathrm{H}_{30}\right)$, retention time 16.00 and trace mass 41 , compound is 1-Pentadecene $\left(\mathrm{C}_{15} \mathrm{H}_{30}\right)$, retention time 16.11 and trace mass 43 , compound is Pentadecane $\left(\mathrm{C}_{15} \mathrm{H}_{32}\right)$, retention time 17.23 and trace mass 41 , compound is 1 -Hexadecene $\left(\mathrm{C}_{16} \mathrm{H}_{32}\right)$, retention time 17.33 and trace mass 57, compound is Hexadecane $\left(\mathrm{C}_{16} \mathrm{H}_{34}\right)$, retention time 18.40 and trace mass 41, compound is E-14-hexadecenal, $\left(\mathrm{C}_{16} \mathrm{H}_{30} \mathrm{O}\right)$, retention time 18.48 and trace mass 57, compound is Heptadecane $\left(\mathrm{C}_{17} \mathrm{H}_{36}\right)$, retention time 19.50 and trace mass 41 , compound is 1-Nonadecene $\left(\mathrm{C}_{19} \mathrm{H}_{38}\right)$, retention time 20.65 and trace mass 57 , compound is Nonadecane $\left(\mathrm{C}_{19} \mathrm{H}_{40}\right)$, retention time 21.65 and trace mass 85 , compound is Eicosane $\left(\mathrm{C}_{20} \mathrm{H}_{42}\right)$, retention time 22.62 and trace mass 57 , compound is Heneicosane $\left(\mathrm{C}_{20} \mathrm{H}_{42}\right)$ etc. In the ultimate phase of the analysis index at retention time 23.54 and trace mass 43, compound is Heneicosane $\left(\mathrm{C}_{20} \mathrm{H}_{42}\right)$, retention time 24.42 and trace mass 85 , and compound is Heneicosane $\left(\mathrm{C}_{20} \mathrm{H}_{42}\right)$ as well, retention time 25.26 and trace mass 57 , compound is Tetracosane $\left(\mathrm{C}_{24} \mathrm{H}_{50}\right)$, retention time 26.09 and trace mass 71 , compound is Heneicosane $\left(\mathrm{C}_{21} \mathrm{H}_{44}\right)$, retention time 26.88 and trace mass 57 , compound is Heneicosane $\left(\mathrm{C}_{21} \mathrm{H}_{44}\right)$, retention time 27.67 and trace mass 57, compound is Octacosane $\left(\mathrm{C}_{28} \mathrm{H}_{58}\right)$, retention time 28.45 and trace mass 57, compound is Octacosane $\left(\mathrm{C}_{28} \mathrm{H}_{58}\right)$, retention time 29.21 and trace mass 57 , compound is Heptacosane $\left(\mathrm{C}_{28} \mathrm{H}_{58}\right)$ and eventually retention time 29.99 and trace mass 57 , compound is same as previous peak that is Heptacosane $\left(\mathrm{C}_{28} \mathrm{H}_{58}\right)$ respectively.

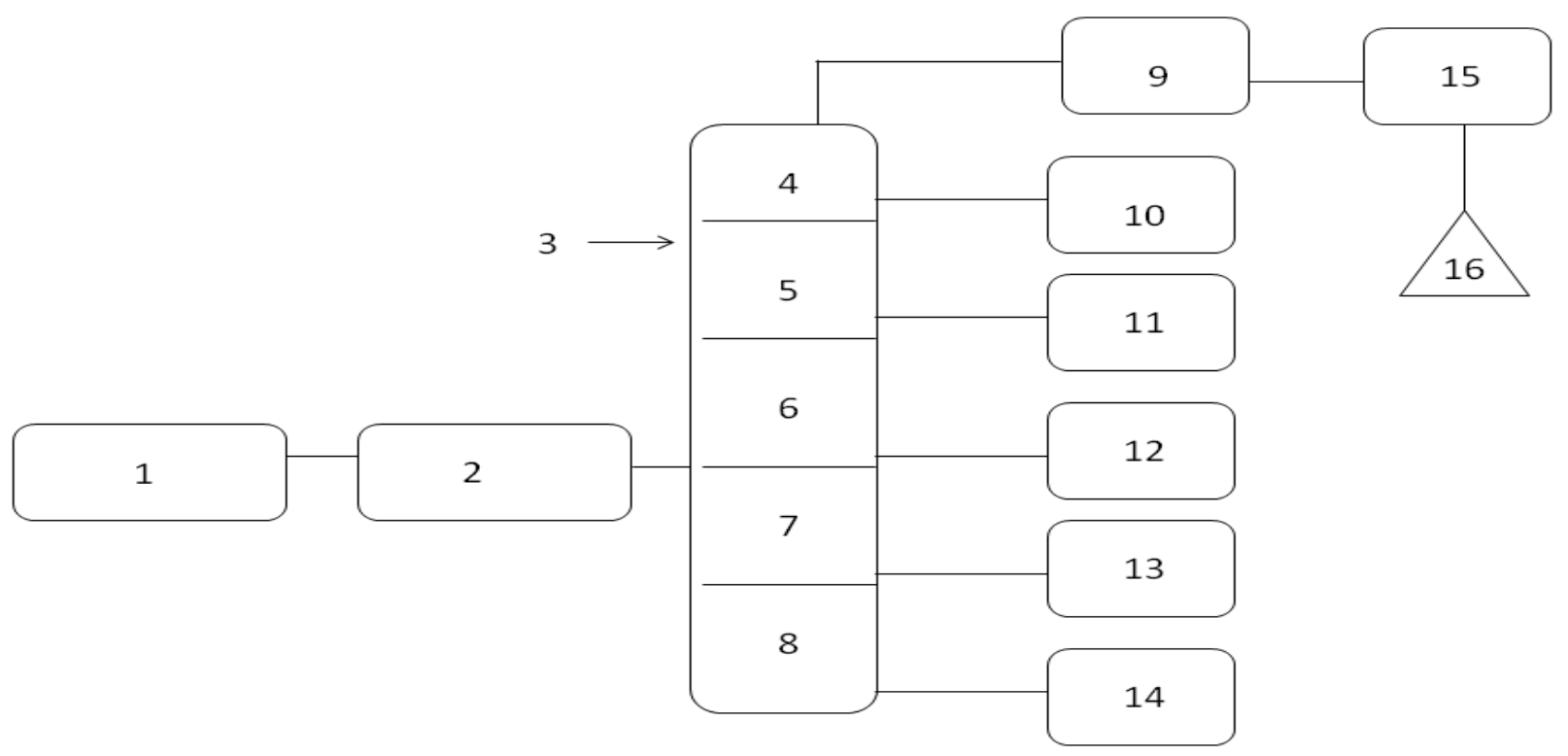

Figure 1. LDPE (Low density polyethylene) waste plast ic to heavy fuel production process diagram 


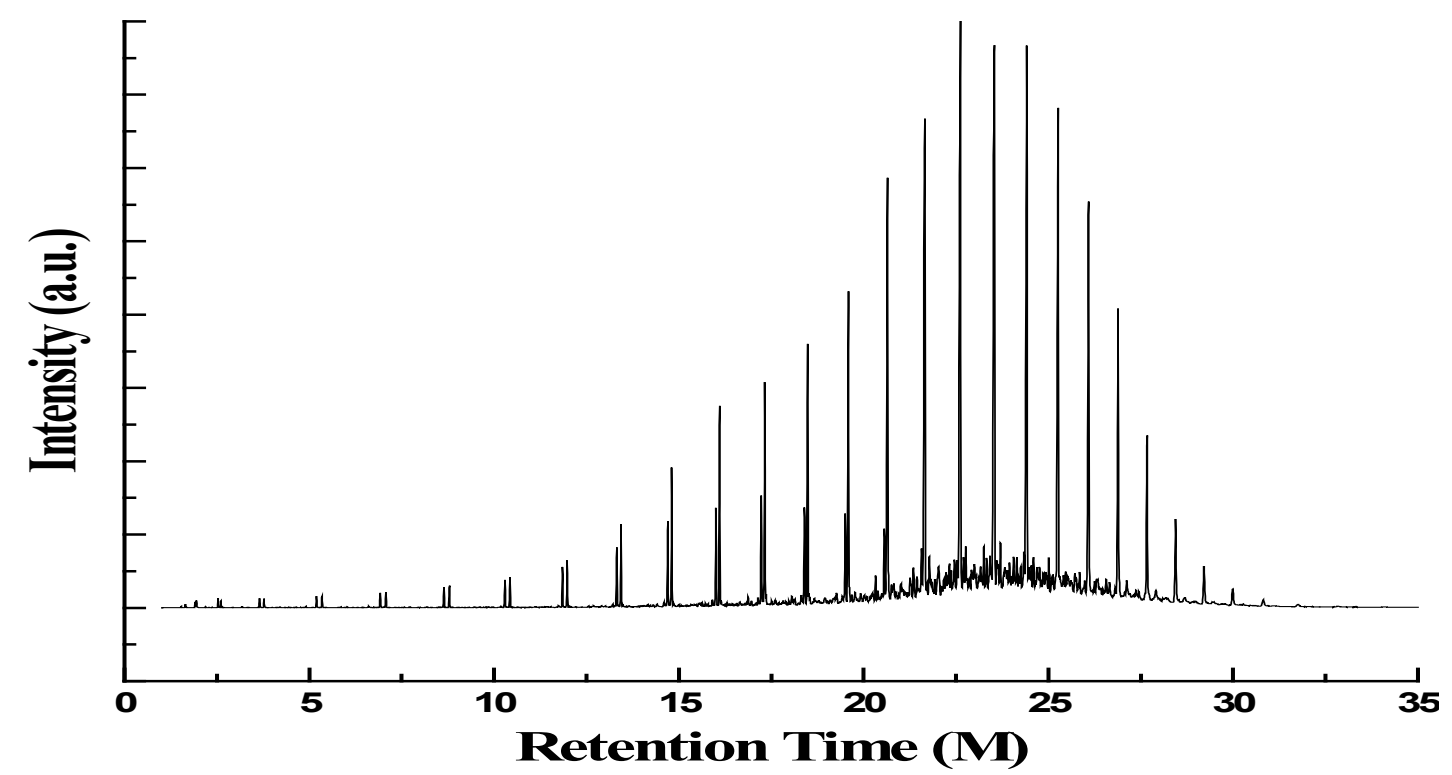

Figure 2. GC/MS (Gas Chromatography and Mass Spectrometer) Chromatogram of LDPE waste plast ic to heavy fuel

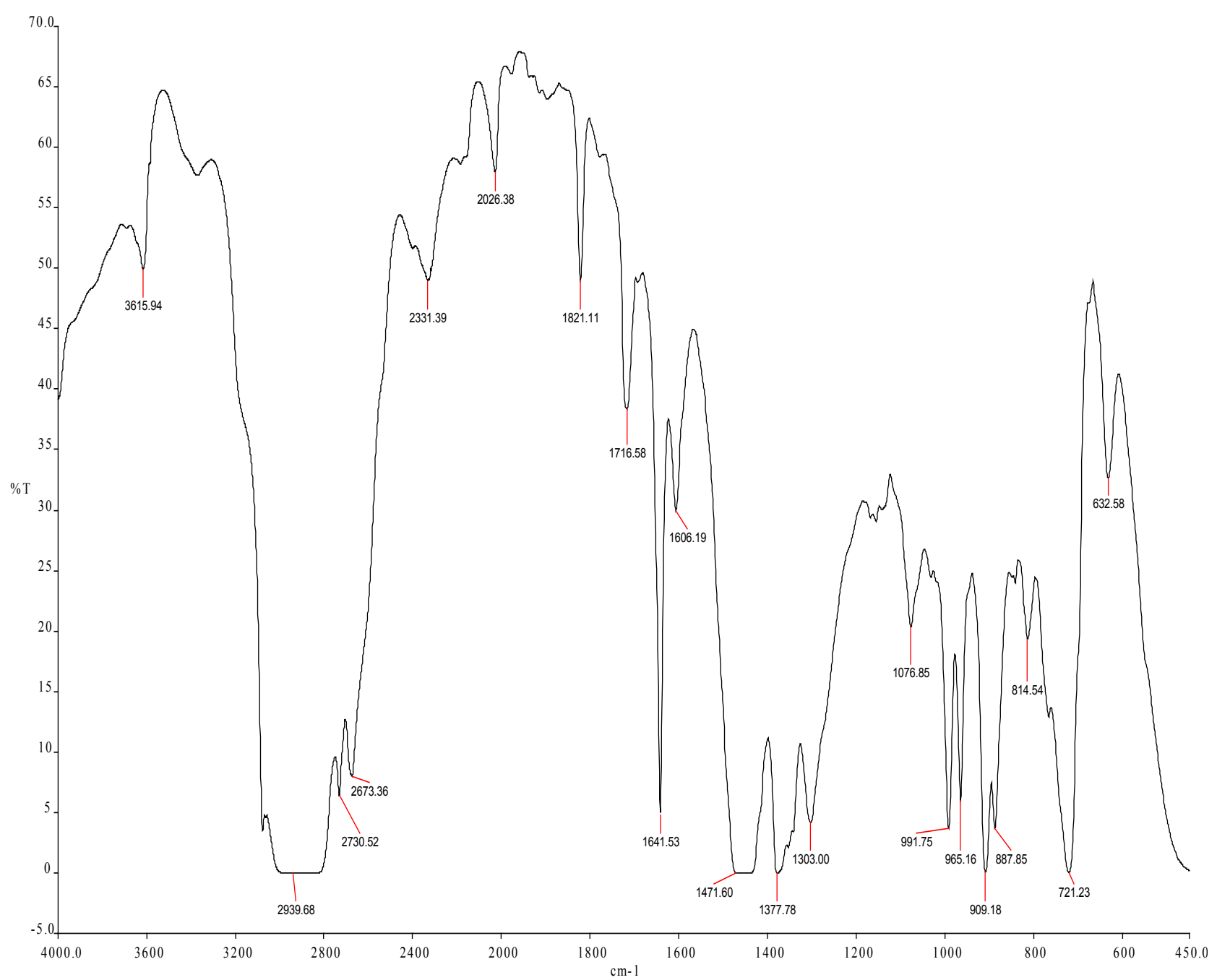

Figure 3. FT-IR (Fourier transform infrared spectroscopy) Spectrum of LDPE waste plast ic to heavy fuel 

into Renewable Heavy Fuel Using Thermal Cracking

Table 1. GC/MS (Gas Chromatography and Mass Spect rometer) Chromatogram of LDPE waste plasticto heavy fuel compound list

\begin{tabular}{|c|c|c|c|c|c|c|}
\hline $\begin{array}{c}\text { Number of } \\
\text { Peak }\end{array}$ & $\begin{array}{c}\text { Retention Time } \\
\text { (min.) }\end{array}$ & $\begin{array}{l}\text { Trace } \\
\text { Mass } \\
(\mathbf{m} / \mathbf{z})\end{array}$ & Compound Name & $\begin{array}{l}\text { Compound } \\
\text { Formula }\end{array}$ & $\begin{array}{l}\text { Molecular } \\
\text { Weight }\end{array}$ & $\begin{array}{l}\text { NIST (National Institu te of } \\
\text { Stan dards and Te chnol ogy) } \\
\text { Library Number }\end{array}$ \\
\hline 1 & 1.91 & 42 & Cyclopropane, ethyl- & $\mathrm{C}_{5} \mathrm{H}_{10}$ & 70 & 114410 \\
\hline 2 & 1.95 & 43 & Pentane & $\mathrm{C}_{5} \mathrm{H}_{12}$ & 72 & 114462 \\
\hline 3 & 2.53 & 41 & $\begin{array}{c}\text { Cyclopropane, } \\
\text { 1-ethyl-2-methyl-, cis- }\end{array}$ & $\mathrm{C}_{6} \mathrm{H}_{12}$ & 84 & 113658 \\
\hline 4 & 2.61 & 41 & Hexane & $\mathrm{C}_{6} \mathrm{H}_{14}$ & 86 & 61280 \\
\hline 5 & 3.65 & 41 & 1-Heptene & $\mathrm{C}_{7} \mathrm{H}_{14}$ & 98 & 107734 \\
\hline 6 & 3.78 & 41 & Heptane & $\mathrm{C}_{7} \mathrm{H}_{16}$ & 100 & 61276 \\
\hline 7 & 5.19 & 41 & 1-Octene & $\mathrm{C}_{8} \mathrm{H}_{16}$ & 112 & 1604 \\
\hline 8 & 5.35 & 43 & Octane & $\mathrm{C}_{8} \mathrm{H}_{18}$ & 114 & 229407 \\
\hline 9 & 6.92 & 41 & 1-Nonene & $\mathrm{C}_{9} \mathrm{H}_{18}$ & 126 & 107756 \\
\hline 10 & 7.07 & 43 & Nonane & $\mathrm{C}_{9} \mathrm{H}_{20}$ & 128 & 228006 \\
\hline 11 & 8.65 & 41 & 1-Decene & $\mathrm{C}_{10} \mathrm{H}_{20}$ & 140 & 118883 \\
\hline 12 & 8.79 & 43 & Decane & $\mathrm{C}_{10} 0^{\mathrm{H}_{22}}$ & 142 & 114147 \\
\hline 13 & 10.30 & 41 & 1-Undecene & $\mathrm{C}_{11} \mathrm{H}_{22}$ & 154 & 5022 \\
\hline 14 & 10.43 & 43 & Undecane & $\mathrm{C}_{11} \mathrm{H}_{24}$ & 156 & 107774 \\
\hline 15 & 11.85 & 41 & 1-Decene & $\mathrm{C}_{10} \mathrm{H}_{20}$ & 140 & 118883 \\
\hline 16 & 11.98 & 43 & Dodecane & $\mathrm{C}_{12} \mathrm{H}_{26}$ & 170 & 291499 \\
\hline 17 & 13.32 & 41 & 1-Tridecene & $\mathrm{C}_{13} \mathrm{H}_{26}$ & 182 & 34733 \\
\hline 18 & 13.43 & 43 & Tridecane & $\mathrm{C}_{13} \mathrm{H}_{28}$ & 184 & 114282 \\
\hline 19 & 14.70 & 41 & 1-Tetradecene & $\mathrm{C}_{14} \mathrm{H}_{28}$ & 196 & 34720 \\
\hline 20 & 14.81 & 43 & Tetradecane & $\mathrm{C}_{14} \mathrm{H}_{30}$ & 198 & 113925 \\
\hline 21 & 16.00 & 41 & 1-Pentadecene & $\mathrm{C}_{15} \mathrm{H}_{30}$ & 210 & 69726 \\
\hline 22 & 16.11 & 43 & Pentadecane & $\mathrm{C}_{15} \mathrm{H}_{32}$ & 212 & 107761 \\
\hline 23 & 17.23 & 41 & 1-Hexadecene & $\mathrm{C}_{16} \mathrm{H}_{32}$ & 224 & 69727 \\
\hline 24 & 17.33 & 57 & Hexadecane & $\mathrm{C}_{16} 6^{\mathrm{H}_{34}}$ & 226 & 114191 \\
\hline 25 & 18.40 & 41 & E-14-Hexadecenal & $\mathrm{C}_{16} \mathrm{H}_{30} \mathrm{O}$ & 238 & 130980 \\
\hline 26 & 18.48 & 57 & Heptadecane & $\mathrm{C}_{17} \mathrm{H}_{36}$ & 240 & 107308 \\
\hline 27 & 19.50 & 41 & 1-Nonadecene & $\mathrm{C}_{19}{ } \mathrm{H}_{38}$ & 266 & 113626 \\
\hline 28 & 19.59 & 57 & Nonadecane & $\mathrm{C}_{19} \mathrm{H}_{40}$ & 268 & 114098 \\
\hline 29 & 20.56 & 41 & 1-Nonadecene & $\mathrm{C}_{19}{ } \mathrm{H}_{38}$ & 266 & 113626 \\
\hline 30 & 20.65 & 57 & Nonadecane & $\mathrm{C}_{19}{ } \mathrm{H}_{40}$ & 268 & 114098 \\
\hline 31 & 21.65 & 85 & Eicosane & $\mathrm{C}_{20} \mathrm{H}_{42}$ & 282 & 290513 \\
\hline 32 & 22.62 & 57 & Heneicosane & $\mathrm{C}_{21} \mathrm{H}_{44}$ & 296 & 107569 \\
\hline 33 & 23.54 & 43 & Heneicosane & $\mathrm{C}_{21} \mathrm{H}_{44}$ & 296 & 107569 \\
\hline 34 & 24.42 & 85 & Heneicosane & $\mathrm{C}_{21} \mathrm{H}_{44}$ & 296 & 107569 \\
\hline 35 & 25.26 & 57 & Tetracosane & $\mathrm{C}_{24} \mathrm{H}_{50}$ & 338 & 248196 \\
\hline 36 & 26.09 & 71 & Heneicosan & $\mathrm{C}_{21} \mathrm{H}_{44}$ & 296 & 107569 \\
\hline 37 & 26.88 & 57 & Heneicosane & $\mathrm{C}_{21} \mathrm{H}_{44}$ & 296 & 107569 \\
\hline 38 & 27.67 & 57 & Octacosane & $\mathrm{C}_{28} \mathrm{H}_{58}$ & 394 & 134306 \\
\hline 39 & 28.45 & 57 & Octacosane & $\mathrm{C}_{28} \mathrm{H}_{58}$ & 394 & 134306 \\
\hline 40 & 29.21 & 57 & Heptacosane & $\mathrm{C}_{27} \mathrm{H}_{56}$ & 380 & 79427 \\
\hline 41 & 29.99 & 57 & Heptacosane & $\mathrm{C}_{27} \mathrm{H}_{56}$ & 380 & 79427 \\
\hline
\end{tabular}

Table 2. LDPE (Low density polyethylene) waste plastic to heavy fuel functional group name

\begin{tabular}{cccccc}
\hline Number of Wave & $\begin{array}{c}\text { Wave Number } \\
\left(\mathbf{c m}^{-1}\right)\end{array}$ & $\begin{array}{c}\text { Functional Group } \\
\text { Name }\end{array}$ & Number of Wave & $\begin{array}{c}\text { Wave Number } \\
\left(\mathbf{c m}^{-1}\right)\end{array}$ & $\begin{array}{c}\text { Functional Group } \\
\text { Name }\end{array}$ \\
\hline 1 & 3615.94 & Free $\mathrm{OH}$ & 11 & 1471.60 & $\mathrm{CH}_{3}$ \\
2 & 2939.68 & $\mathrm{C}-\mathrm{CH}_{3}$ & 12 & 1377.78 & $\mathrm{CH}_{3}$ \\
3 & 2730.52 & $\mathrm{C}-\mathrm{CH}_{3}$ & 15 & 991.75 & $-\mathrm{CH}=\mathrm{CH}_{2}$ \\
4 & 2673.36 & $\mathrm{C}-\mathrm{CH}_{3}$ & 16 & 965.16 & $-\mathrm{CH}=\mathrm{CH}-(\mathrm{cis})$ \\
7 & 1821.11 & Non-Conjugated & 17 & 909.18 & $-\mathrm{CH}=\mathrm{CH}_{2}$ \\
8 & 1716.58 & Non-Conjugated & 18 & 887.85 & $\mathrm{C}=\mathrm{CH}_{2}$ \\
9 & 1641.53 & Non-Conjugated & 20 & 721.23 & $-\mathrm{CH}=\mathrm{CH}-(\mathrm{cis})$ \\
\hline
\end{tabular}


Perkin Elmer FT-IR (Fourier transform infrared spectroscopy, Spectrum 100) analysis of LDPE waste plastic to heavy fuel oil (Figure 3 and Table 2) shows the following types of functional groups starting at wave number $3615.94 \mathrm{~cm}^{-1}$, derived functional group is Free $\mathrm{OH}$, wave nu mber $2939.68 \mathrm{~cm}^{-1}, 2730.52 \mathrm{~cm}^{-1}$ and $2673.36 \mathrm{~cm}^{-1}$ functional group is $\mathrm{C}-\mathrm{CH}_{3}$, wave number $1821.11 \mathrm{~cm}^{-1}$, $1716.58 \mathrm{~cm}^{-1}$, and $1641.53 \mathrm{~cm}^{-1}$ functional group is Non-Conjugated, wave number $1606.19 \mathrm{~cm}^{-1}$ functional group is Conjugated. As well as wave number $1471.60 \mathrm{~cm}^{-1}$ and $1377.78 \mathrm{~cm}^{-1}$ functional group is $\mathrm{CH}_{3}$, wave number $991.75 \mathrm{~cm}^{-1}$ and $909.18 \mathrm{~cm}^{-1}$, functional group is $-\mathrm{CH}=\mathrm{CH}_{2}$ and ultimately wave number $887.85 \mathrm{~cm}^{-1}$ functional group is $\mathrm{C}=\mathrm{CH}_{2}$ and wave number $721.33 \mathrm{~cm}^{-1}$ functional group is $-\mathrm{CH}=\mathrm{CH}$-(cis). Energy values are calculated, using formula is $\mathrm{E}=\mathrm{h} v$, Where $\mathrm{h}=\mathrm{Planks}$ Constant, $\mathrm{h}=6.626 \times 10^{-34} \mathrm{~J}, \mathrm{v}=$ Frequency in Hertz $\left(\mathrm{sec}^{-1}\right)$, Where $v=\mathrm{c} / \lambda, \mathrm{c}=$ Speed of light, where, $\mathrm{c}=3 \times 10^{10} \mathrm{~m} / \mathrm{s}, \mathrm{W}=1 / \lambda$, where $\lambda$ is wave length and $\mathrm{W}$ is wave number in $\mathrm{cm}^{-1}$. Therefore the equation $\mathrm{E}=\mathrm{hv}$, can substitute by the following equation, $\mathrm{E}=\mathrm{hcW}$. All functional group band energy is reflecting calorific value. According to their wave number such as for $3615.94\left(\mathrm{~cm}^{-1}\right)$ calculated energy, $E=7.18 \times 10^{-20}$ J. Similarly, wave number 2939.68 $\left(\mathrm{cm}^{-1}\right)$ calculated energy, $\mathrm{E}=5.83 \times 10^{-20} \mathrm{~J}$, wave number $2730.52\left(\mathrm{~cm}^{-1}\right)$ calculated energy, $E=5.42 \times 10^{-20} \mathrm{~J}$, wave number $2673.36\left(\mathrm{~cm}^{-1}\right)$ calculated energy, $E=5.31 \times 10^{-20} \mathrm{~J}$, wave number $1821.11\left(\mathrm{~cm}^{-1}\right)$ calculated energy, $\mathrm{E}=3.61 \times 10^{-20} \mathrm{~J}$, wave number $2730.52\left(\mathrm{~cm}^{-1}\right)$ calculated energy, $\mathrm{E}=5.42 \times 10^{-20} \mathrm{~J}$, wave number $1716.58\left(\mathrm{~cm}^{-1}\right)$ energy, $E=3.40 \times 10^{-20} \mathrm{~J}$, wave number $1471.60\left(\mathrm{~cm}^{-1}\right)$ calculated energy, $\mathrm{E}=2.92 \times 10^{-20} \mathrm{~J}$, wave number 1377.78 $\left(\mathrm{cm}^{-1}\right)$ energy, $\mathrm{E}=2.73 \times 10^{-20} \mathrm{~J}$, wave number $991.75\left(\mathrm{~cm}^{-1}\right)$ calculated energy, $\mathrm{E}=1.97 \times 10^{-20} \mathrm{~J}$, wave number 965.16 $\left(\mathrm{cm}^{-1}\right)$ calculated energy, $\mathrm{E}=1.91 \times 10^{-20} \mathrm{~J}$, wave number $887.85\left(\mathrm{~cm}^{-1}\right)$ calculated energy, $\mathrm{E}=1.80 \times 10^{-20} \mathrm{~J}$, wave number $909.18\left(\mathrm{~cm}^{-1}\right)$ calculated energy, $\mathrm{E}=1.80 \times 10^{-20} \mathrm{~J}$ and eventually wave number $721.23\left(\mathrm{~cm}^{-1}\right)$ calculated energy is , $\mathrm{E}=1.43 \times 10^{-20} \mathrm{~J} 1.97 \times 10^{-20} \mathrm{~J}$ respectively.

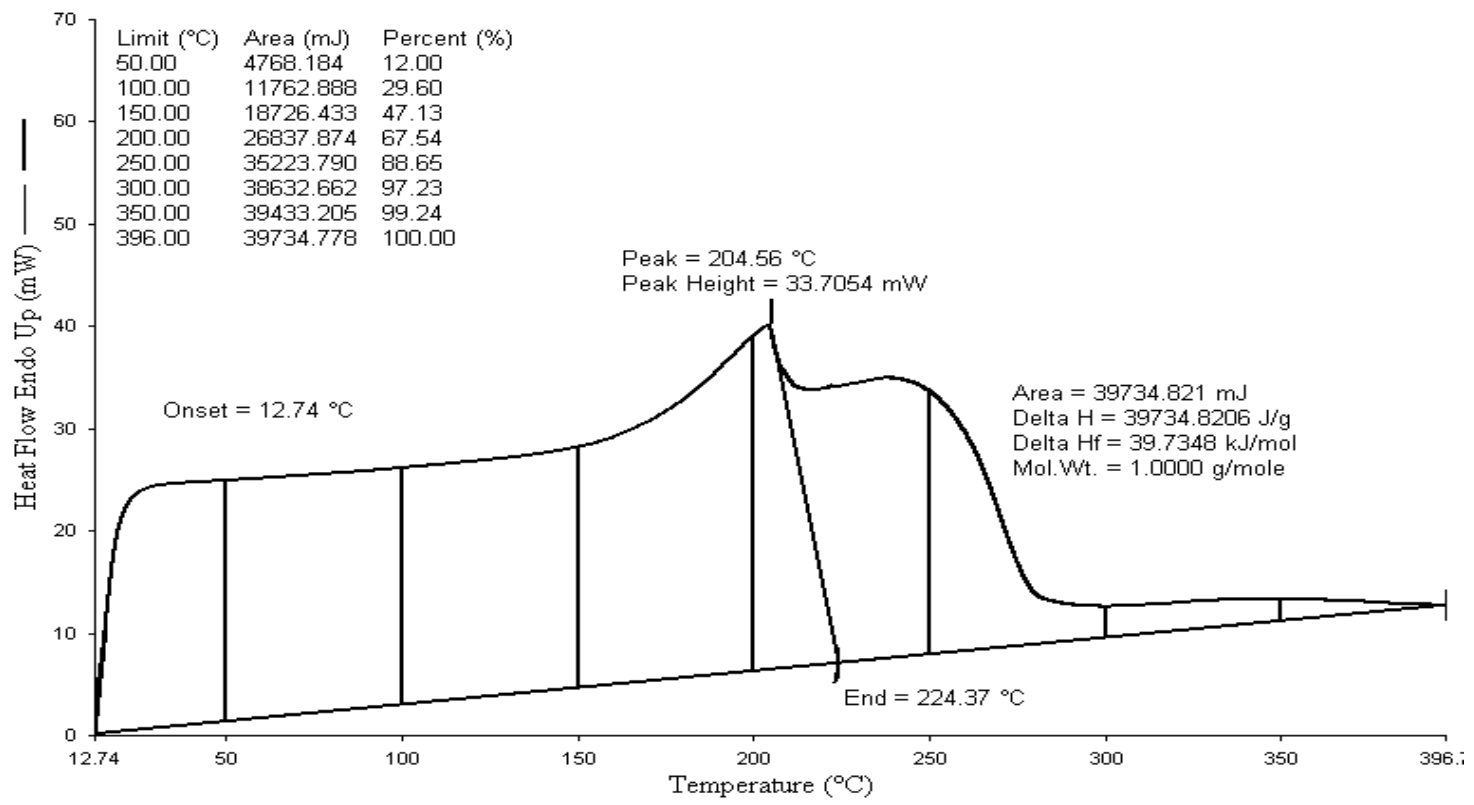

Figure 4. Differential Scanning Calorimeter (DSC) graph of LDPE waste plast ic to heavy fuel

Table 3. Low density polyethylene to solid black residue analysis result by ICP (Induced Coupled Plasma)

\begin{tabular}{|c|c|c|c|c|c|}
\hline $\begin{array}{l}\text { Test Me thod } \\
\text { Name }\end{array}$ & $\begin{array}{l}\text { Trace Me tal } \\
\text { Name }\end{array}$ & $\begin{array}{c}\text { LDPE Waste Plastic } \\
\text { to } \\
\text { Residue (ppm) } \\
\end{array}$ & $\begin{array}{l}\text { Test Me thod } \\
\text { Name }\end{array}$ & $\begin{array}{c}\text { Trace Me tal } \\
\text { Name }\end{array}$ & $\begin{array}{c}\text { LDPE Waste Plastic } \\
\text { to } \\
\text { Residue (ppm) }\end{array}$ \\
\hline \multirow[t]{13}{*}{ ASTM D1976 } & Silver & $<1.0$ & ASTM D1976 & Magnesium & 221.6 \\
\hline & Aluminium & 7806 & & Manganese & 17.8 \\
\hline & Arsenic & 23.6 & & Sodium & 1201 \\
\hline & Boron & 24.3 & & Nickel & 148.7 \\
\hline & Barium & 11.4 & & Lead & 10.8 \\
\hline & Beryllium & $<1.0$ & & Antimony & $<1.0$ \\
\hline & Calcium & 1041 & & Selenium & $<1.0$ \\
\hline & Cadmium & $<1.0$ & & Silicon & 77.0 \\
\hline & Chromium & 29.2 & & Tin & 274.3 \\
\hline & Copper & 82.4 & & Titanium & 191.1 \\
\hline & Iron & 4280 & & Vanadium & 17.0 \\
\hline & Potassium & 106.7 & & Zinc & 1617 \\
\hline & Lithium & $<1.0$ & & & \\
\hline
\end{tabular}


LDPE waste plastic to $5^{\text {th }}$ fractional fuel or heavy fuel was (Figure 4) analysed by DSC (Differential scanning calorimeter) and measured the boiling point temperature and enthalpy value in this fuel. After analysis fuel sample we found onset temperature is $12.74^{\circ} \mathrm{C}$, Peak temperature is $204.56^{\circ} \mathrm{C}$ and peak height is $33.7054 \mathrm{~mW}$. Peak height is represents the heat flow Endo up $40 \%$ from total $100 \%$. The graph showed area is $39734.821 \mathrm{~mJ}$ and enthalpy delta $\mathrm{H}$ value is $39734.8206 \mathrm{~J} / \mathrm{g}$. This produce fuel hydrocarbon compounds are heavier and making heavy fuel we used higher temperature profile in the fractionation process and temperature range was $340-365^{\circ} \mathrm{C}$. Fifth $\left(5^{\text {th }}\right)$ fractional or heavy fuel boiling starts from at $12.74{ }^{\circ} \mathrm{C}$ and end boiling temperature finished almost close to $300{ }^{\circ} \mathrm{C}$, because this fuel has heavier straight long chain hydrocarbon compounds like alkane or alkene co mpounds.

In Table 3 showed trace metal analysis result by using ICP from LDPE waste plastic to heavy fuel production. Solid black residue generated $3 \%$ from total initial feed. This residue was left over because plastic manufacturing company adding different types of additives for plastic hard shape and thickness. This additive was come out or left over after fuel production process and this solid residue have also good Btu (British thermal units) value. ICP (A merican Standard Testing Method) (ASTM) D1976 test result showed that in LDPE waste plastic to residue trace metal content are Aluminium $<1.0 \mathrm{ppm}$ (Parts per million), Arsenic $23.6 \mathrm{ppm}$, Boron $24.3 \mathrm{ppm}$, Barium $11.4 \mathrm{ppm}$, Beryllium $<1.0$ ppm, Calcium 1041 ppm, Cadmium $<1.0$ ppm, Chromium 29.2 ppm, Copper 82.4 ppm, Iron 4280 ppm, Potassium 106.7 ppm, Lith ium $<1.0$ ppm, Silver $<1.0$ ppm, Magnesium $221.6 \mathrm{ppm}$, Manganese $17.8 \mathrm{ppm}$, Sodium $1201 \mathrm{ppm}$, Nickel $148.7 \mathrm{ppm}$, Lead $10.8 \mathrm{ppm}$, Antimony $<1.0 \mathrm{pp}$, Selenium $<1.0 \mathrm{ppm}$, Silicon $77.0 \mathrm{ppm}$, Tin $274.3 \mathrm{ppm}$, Titanium $191.1 \mathrm{ppm}$, Vanadium $17.0 \mathrm{ppm}$, Zinc $1617 \mathrm{ppm}$.

\section{Conclusions}

Thermal cracking with fractional distillation process was performed with LDPE waste plastic and produced fractional heavy fuel without using any kind of catalyst. Two types of temperature were used for this experiment one was liquefaction temperature and another one was fractional distillation column temperature for LDPE waste plastic to heavy fuel generation. Produced fuel was analysis by using various types of equipment such as GC/MS, FT-IR and DSC. GC/MS analysis result indicates that produced fuel has short chain hydrocarbon to long chain hydrocarbon compounds and starting short chain hydrocarbon compound carbon number is $\mathrm{C} 5$ and long chain hydrocarbon compound carbon number is C28. By using FT-IR analysis result showed also produced fuel functional group band energy. LDPE waste plastic to heavy fuel has alkane and alkene group compound which is aliphatic compound because in the initial raw material compound was only straight chain hydrocarbon compounds. Produced fuel could be use all heavy equipment engine or feed for feed stock refinery or feed for electricity generation. By using this established technology could be solve the LDPE waste plastic related environmental problem and boost up the renewable energy generation from LDPE waste plastic.

\section{ACKNOWLEDGEMENTS}

The authors acknowledge the support (Financial) of Dr. Karin Kaufman, the founder and sole owner of Natural State Research, Inc. The authors also acknowledge the valuable contributions NSR (Natural State Research) laboratory team members during the preparation of the manuscript.

\section{REFERENCES}

[1] Y. Sakata, M.A. Uddin, A. Muto, K. Koizumi, M. Narazaki, K. Murata, M. Kaiji, Polym. Recycling, 2 (1996) 309.

[2] Aguado J, Serrano D. In: Clark JH, editor. RSC clean technology monographs, on feedstock recycling of waste plastic. Cambridge: Royal Society of Chemistry; (1999).

[3] Saunders DC. Technical workshop report on sustainable management for plastics with bromine in Tokyo; (1999).

[4] Y. Sakata, M.A. Uddin, A. Muto, K. Koizumi, M. Narazaki, K. Murata, M. Kaiji, Polym. Recycling 2 (1996) 309.

[5] L. Tange, BSEF Technical Workshop on Sustainable Management for Plastics with Bromine, Tokyo, 19 November, (1999).

[6] W. Kaminsky, F. Hartmann, Angew. Chem. Int. Ed. 39 (2000) 331 .

[7] J. Walendziewski, Fuel 81 (2002) 473.

[8] R. Miranda, H. Pakdel, C. Roy, C. Vasile, Polym. Degrad. Stab. 73 (2001) 47.

[9] M. Blazso, E. Jakab, J. Anal. Appl. Pyrol. 49 (1999) 125.

[10] H. Bockhorn, A. Hornung, U. Hornung, P. Jakobstroer, M. Kraus, J. Anal. Appl. Pyrol. 49 (1999) 97.

[11] M. A Uddin, K. Koizumi, K. Murata, Y. Sakata, Polym. Degrad. Stab. 56 (1997) 37.

[12] Y. Sakata, M.A. Uddin, A. Muto, M. Narazaki, K. Koizumi, K. Murata, K. Mitsuo, Ind. Eng. Chem. Res. 37 (1998) 2889.

[13] S. Ali, A.A. Garforth, D.H. Harris, D.J. Rawlence, Y. Uemichi, Catal. Today 75 (2002) 247.

[14] A. Pifer, A. Sen, Angew. Chem. Int. Ed. 37 (1998) 3306.

[15] Thallada Bhaskar, Jun Kaneko , Akinori Muto, Yusaku Sakata, Emma Jakab, Toshiki Matsui, Md. Azhar Uddin. Effect of poly (ethyleneterephthalate) on the pyrolysis of brominated flame retardant containing high impact 
polystyrene and catalytic debromination of the liquid products. J. Anal. Appl. Pyroly sis 71 (2004) 765-777.

[16] Mihai Brebua, Thallada Bhaskar, Kazuya Murai, Akinori Muto, Yusaku Sakata, Md. Azhar Uddin, The effect of PVC and/or PET on thermal degradation of polymer mixtures containing brominated ABS, Fuel 83 (2004) 2021-2028.

[17] McNeill IC, Memetea L, Mohammed MH, Fernandes AR, Ambidge P. Polym Degrad Stab (1998);62:145.
[18] Borojovich EJC, Aizenshtat Z. J Anal App1 Pyrolysis (2002); 63:105. See also page 129 .

[19] Brebu M, Uddin MA, Muto A, Sakata Y, Vasile C. J Anal Appl Py roly sis (2002);63(1):43.

[20] Brebu M, Uddin MA, Muto A, Sakata Y, Vasile C. Energy Fuels $(2001) ; 15(3): 559$. 